\title{
$1 / f$ noise in the intensity fluctuations of vertical-cavity surface-emitting lasers subject to parallel optical injection
}

\author{
Miguel A. Rodríguez, ${ }^{1}$ Florian Denis-le Coarer, ${ }^{2}$ and Ángel Valle ${ }^{1}$ \\ ${ }^{1}$ Instituto de Física de Cantabria (IFCA), CSIC-UNICAN, E-39005 Santander, Spain \\ ${ }^{2}$ OPTEL Research Group, LMOPS Laboratory, CentraleSupélec, Université de Paris-Saclay and Université de Lorraine, 57070 Metz, France
}

(Received 6 November 2017; revised manuscript received 7 March 2018; published 6 April 2018)

\begin{abstract}
A first analysis of fluctuations of the light intensity of vertical-cavity surface-emitting lasers operating in a bistable regime reveals the presence of $1 / f$ noise. In this regime the intensity fluctuates between two recently characterized states with residence times $\left\{\tau_{1}\right\}$ and $\left\{\tau_{2}\right\}$. We identify three distinct processes. One of them presents a coherence enhancement phenomenon, and in the other two the distribution of residence times in one of the states follows either a power law $P\left(\tau_{1}\right) \sim \tau_{1}^{-2}$ or $P\left(\tau_{2}\right) \sim \tau_{2}^{-2}$, and this is the cause of the $1 / f$ shape in the spectral density of the intensity. The process at the coherence enhancement zone shows $1 / f$ fluctuations in the light intensity and also in the time residence process. It is shown that the origin of these fluctuations is due to a power-law distribution in the time separation between pulses observed in the time residence series.
\end{abstract}

DOI: 10.1103/PhysRevE.97.042105

\section{INTRODUCTION}

Experiments with vertical-cavity surface-emitting lasers (VCSELs) subject to optical injection exhibit a rich dynamics that has been the subject of interesting studies of stochastic nonlinear processes [1]. By injecting linearly polarized light from an external laser, either in the direction of the linear polarization emitted by the VCSEL (parallel) or in its orthogonal direction, a rich variety of states exhibiting harmonic, periodic, and chaotic dynamics can be observed by tuning some external parameters such as the injected power and the detuning frequency. Among these states, there are two that have attracted considerable attention of researchers due to their possible utility in optical communications. Semiconductor lasers subject to parallel optical injection are used in modern optical communications. The first state corresponds to the period-1 (P1) oscillations that are generated in these systems. These oscillations are used as photonic microwave sources in radioover-fiber (ROF) optical communication systems [2,3]. The main advantage of the P1 state is that it can be used for optical single sideband modulation [3]. This type of modulation is employed to reduce the periodic fading of the radiofrequency signals caused by the chromatic dispersion of the optical fibers [2]. The second state occurs when the orthogonal polarization mode is activated [polarization switching (PS)] and the VCSEL locks its frequency to that of the injection laser [injection locking (IL)]. Usually only one of these phenomena occurs, but in a recent work [4] using parallel optical injection, a new state in which both phenomena (IL+PS) occur simultaneously was experimentally observed and theoretically analyzed. In IL+PS, injection locking of the parallel polarization and excitation of the free-running orthogonal polarization are simultaneously observed. The fluctuation dynamics of this new state, when (in a parameter space) it is on the frontier with other states, presents interesting features [5-7].

Here we focus on the case in which bistability occurs between the (IL+PS), which is a fixed point, and periodic (P1) states in the frontier of Hopf bifurcation [6]. The fluctuations generated by a hopping process between these states exhibit two unusual properties. First, there is a central zone of normal bistability in the parameter space where the time residence distribution of both states follows a standard (not heavy tail) statistics. Assuming that fluctuations are driven by external noise, we can speak of a phenomenon of coherence enhancement (not coherence resonance), since we have no control over the noise intensity [8]. The unusual facts are the $1 / f$ fluctuations observed in the light intensity and in the residence time processes. Second, close to the limit between this zone and the zones of stability of the P1 and IL+PS states, we find a kind of intermittent behavior where the residence time distribution of one of the mentioned states is a power law with an exponent close to -2 , and where fluctuations of the light intensity are characterized by a power spectrum close to $1 / f$ with peaked structure.

The existence of $1 / f^{\beta}$ noise in hopping processes was first studied in nonlinear oscillators [9]. It is interesting because a standard hopping process with well-defined mean residence times between states should exhibit Lorentzian power $\operatorname{spectra}\left(\sim 1 / f^{2}\right)$ [10]. The existence of $1 / f^{\beta}$ spectra with $\beta$ close to 1 thus means that some unusual mechanism is present in the hopping process. Any one of three generic mechanisms could explain this situation: (i) Hopping is not between two or more attractors but between two loci of the same attractor. The normal noisy process that produces hopping between attractors is substituted here by a chaotic process (deterministic diffusion) [9-11]. (ii) Hopping is between a set of attractors (multistability) with a given distribution of residence time processes [12,13]. (iii) Hopping is between two attractors submitted to external fluctuations that change the escape properties of the attractors $[14,15]$. In our experiment, there are two involved states that are not chaotic, a limit cycle (P1) and a fixed point (IL+PS), so only the type (iii) mechanism is valid to explain the observed anomalous behavior. 
Usually the identification of the exact physical mechanism producing the $1 / f$ noise is complicated, but at least one can determine its generic properties. A generic mechanism of $1 / f$ production applicable to our process was first introduced by Manneville [16] in the context of intermittency of chaos and turbulence. In a more general context, this result suggests that in any multilevel fluctuating signal with intermittent behavior, the power-law exponent of its residence time distribution is connected with its spectral exponent. This connection has been found in many systems, ranging from other intermittent chaotic systems [17], blinking quantum dots [18,19], liquid crystals [20], and nanoscale electrodes [21]. A kind of intermittent behavior, called two-state on-off intermittency, has been found in several types of lasers: distributed-feedback semiconductor lasers (DFBs) [22], diode lasers with an external cavity [15], optical injected dual-mode semiconductor lasers [23], and mutually coupled diode lasers [24]. However, only a characterization of the time residence distribution of the laminar state with an exponent close to $-3 / 2$ has been reported in these studies, without connecting with spectral properties. Note that our experiment can be seen as a type of on-off intermittency where the laminar (off) state is one of the involved states (P1) or (IL+PS). In all these processes, a proper spectral analysis requires special treatment, since there is a lack of ergodicity $[19,25]$ and the standard spectral density is not well defined. This treatment has been developed only in recent times and with synthetic signals [26-29]. In this paper, we use one of these techniques to analyze and classify the $1 / f$ noise found in our experiments with VCSELs.

The organization of the paper is as follows. In Sec. II a background of the methods used in the analysis of fluctuations is presented. In Sec. III a brief sketch of the experimental setup and results is shown. In Sec. IV the $1 / f$ signal observed in cases with intermittent behavior is analyzed following our previous results. In Sec. V the analysis is devoted to fluctuations of the time residence series in the coherence enhancement process. Finally, conclusions are presented in Sec. VI.

\section{ANALYSIS OF FLUCTUATIONS}

A standard method to analyze fluctuations of a stationary signal is the use of power spectrum densities (PSDs). Typical spectra consist of a set of peaks superimposed on a horizontal background line of frequencies indicating the presence of a true signal in a noisy environment. When this line is not horizontal but a power such as $1 / f^{\beta}$ appears, a more careful analysis should be used. Stationarity and/or ergodicity of the signal should be questioned [25], and other methods were used throughout the past 50 years to solve this problem.

In general the analysis of complex signals has been performed using either a direct or an indirect treatment of fluctuations. Direct methods mainly include the use of widths (detrended fluctuation analysis [31]), correlations [27], and PSDs [26]. Indirect methods comprise a wide set of possibilities, ranging from the use of entropy measures [32], wavelets [33], or even complex networks [34]. We are going to use a recently proposed method that can be used to treat this problem by adapting the existing spectral methods [26]. We have two clear advantages with this choice. One is the use of a standard method in the analysis of optical signals. The other is the existence of a classification of $1 / f$ processes based on this method that gives useful additional information.

\section{A. Complete spectral scaling}

In recent years, it has been shown how to treat this problem by adapting the existing spectral methods. One should simply consider the size of the signal $T$ as a relevant variable and perform a complete spectral analysis in which both the scaling of frequencies and sizes, $T^{\gamma} / f^{\beta}$, are taken into account $[26,30]$. It is a way of adapting the Wiener-Kinchine theorem to nonstationary or nonergodic situations [27-29].

The basis of these methods is related to the necessity of accounting for local and global structural properties in a general time series [26]. Consider a signal given by a time series, $Y(t) \equiv\left\{y_{t_{1}}, y_{t_{2}}, \ldots, y_{t_{N}=T}\right\}$, representing one sample of an ensemble, $\left\{Y_{T}\right\}$, of a numerical or empirical process of size $N$. Global properties are related to affine transformations. Let us consider series with constant increments, $t_{i+1}-t_{i}=\Delta$, and take affine transformations such as $\Delta^{\prime}=\lambda \Delta, y^{\prime}=\Delta^{\theta} y$. A global exponent, $\alpha$, can be defined if, in the transformation, it keeps the statistical description of the series invariant, $Y_{\lambda T}(\lambda t) \sim \lambda^{-\alpha} Y_{T}(t)$. On the other hand, local properties correspond to changes in the number of points of the series, keeping the time increment constant. As in the global case, a local exponent $\alpha_{\mathrm{loc}}$ can be defined if $Y_{\lambda T}(t) \sim \lambda^{-\alpha_{\mathrm{loc}}} Y_{T}$. It is worth remarking that at least two distinct exponents, accounting for their local and global character, are necessary for a proper description of time-series scaling. Only the self-affine case, where $\alpha_{\mathrm{loc}}=\alpha$, requires only one exponent.

To quantify the global behavior, one takes the scaling exponents of the asymptotic variation of moments with $T$, as, for instance, the mean value $\left\langle\overline{Y_{T}}\right\rangle \sim T^{v}$ and variance $\left\langle\overline{\left(Y_{T}-\overline{Y_{T}}\right)^{2}}\right\rangle \sim T^{2 \alpha}$. From now on, $\overline{\#}$ and $\langle \#\rangle$ denote time and sample averaging, respectively. For measuring local properties, a variety of methods going from spectral to wavelet analysis were used in the literature. Here we use spectral methods. The spectral density of a sample is defined by $S_{T}(f)=$ $\frac{1}{T} \widehat{Y_{T}(f)} \widehat{Y_{T}(-f)}$, where $\widehat{\#}$ is a Fourier transform. Ensemble averages $S(f, T)=\left\langle S_{T}(f)\right\rangle$ are taken with the aim of reducing spectral fluctuations. Then we identify the local exponent with the spectral exponent $\alpha_{s}$ given by $S(f, T) \sim 1 / f^{2 \alpha_{s}+1}$. If we only consider typical time series that are either symmetric with respect to their mean values $(v=0)$ or completely asymmetric, $v=\alpha$, we can conclude that only two independent exponents, $\alpha$ and $\alpha_{s}$, are necessary to have a proper characterization of time series based on spectral properties [26]. And these exponents could be directly obtained from power spectra of series with variable size, since a complete scaling of power spectra is written as $[26,30]$

$$
S(f, T) \sim \begin{cases}\frac{T^{2\left(\alpha-\alpha_{s}\right)}}{f^{2 \alpha_{s}+1}} & \text { if } \alpha_{s} \geqslant 0,\left(\alpha_{s}, \alpha\right) \neq(0,0), \\ \frac{[\log (T)]^{-1}}{f} & \text { if } \alpha_{s}=0, \alpha=0, \\ \frac{T^{2 \alpha}}{f^{2 \alpha_{s}+1}} & \text { if } \alpha_{s}<0 .\end{cases}
$$

This scaling allows us to classify time series into classes using the two exponents $\alpha_{s}$ and $\alpha$. These classes represent distinct types of series that share some geometrical property. Concerning classes producing $1 / f$ signals, it is observed that 
up to four classes produce the same standard spectrum [30]. These are the class of self-affine curves (SA) defined by $\alpha_{s}=$ $\alpha>0$, of stationary noise (SN) defined by $\alpha_{s}<0, \alpha=0$, of pure $1 / f$ noise defined by $\alpha_{s}=0, \alpha<0$, and the class of stationary fractal curves (SF) defined by $\alpha_{s}>0, \alpha=0$. A more complete description and mode of generation in each case can be found in [30].

\section{B. Uncorrelated pulses}

Many of the fluctuating signals produced by electronic and photonic devices are in the form of a time series of pulses. Hopping processes are included in these signals.

Let us first consider the case in which the interpulse times $\left\{\tau_{i}\right\}$ are independent variables following a given probability density $P(\tau)$, that is, we have a renewal process. The corresponding time series is written as

$$
Y(t)=\sum_{l} h\left(t-t_{l}, \theta_{l}\right),
$$

where $h(s, \theta)$ is the shape of the pulse centered in $s=0, t_{l}=$ $\sum_{i=1}^{l} \tau_{i}$ are the times at which the pulse is placed, and $\theta$ is a parameter quantifying the shape of the pulse. Calculation of the spectral density gives

$$
S_{T}(f)=\frac{1}{T} \sum_{l, m} \exp \left[-i f\left(t_{l}-t_{m}\right)\right] \tilde{h}\left(f, \theta_{l}\right) \tilde{h}\left(-f, \theta_{m}\right) .
$$

Assuming now that the finite Fourier transform is independent of the position $t_{l}$ and integration range $T$, and that the renewal times $\tau_{l}=t_{l+1}-t_{l}$ and shape parameters $\theta_{l}$ are uncorrelated, we have

$$
S(f, T) \sim \frac{N(T)}{T}\left\langle|\widetilde{h}(f, \theta)|^{2}\right\rangle,
$$

where we have ignored the of-diagonal $(l \neq m)$ terms, which means that the coefficient $\exp \left[-i f\left(t_{l}-t_{m}\right)\right]$ acts as a random phase [25]. $N(T)$ is the mean number of pulses in the interval $T$. When the mean renewal time $\bar{\tau}$ is well defined and finite, $N(T)=\frac{T}{\bar{\tau}}$, the size $T$ is irrelevant in the spectra and we have a case with single scaling. The spectral exponent is exclusively determined by the shape and distribution of pulses. As an example, if the shape of pulses scales as $h(s, \theta) \sim \theta^{-a} g(s / \theta)$ and they are distributed as a power law with $P(z \theta) \sim z^{-b} P(\theta)$, the spectral exponent becomes

$$
\alpha_{s}=1-a-\frac{b}{2} \text {. }
$$

On the other hand, if the mean renewal time is dependent on $T$, we have a case of double scaling in the spectra. As an example, we consider the case with a power law in the probability of waiting times $P(\tau)=(d-1) \tau^{-d}$ with $1<d<2$, $\tau \in(1, \infty)$, where $\bar{\tau}=\frac{d-1}{2-d}\left(T^{2-d}-1\right)$. In this case, using (1), we have

$$
2\left(\alpha-\alpha_{s}\right)=d-2 .
$$

In our experiments, we deal with hopping processes that are, in fact, a series of flat pulses, so our interest is focused on this type of signal. The interpulse time in a hopping process coincides with the so-called residence time, this last notation being more illustrative. In a more general form, we can consider
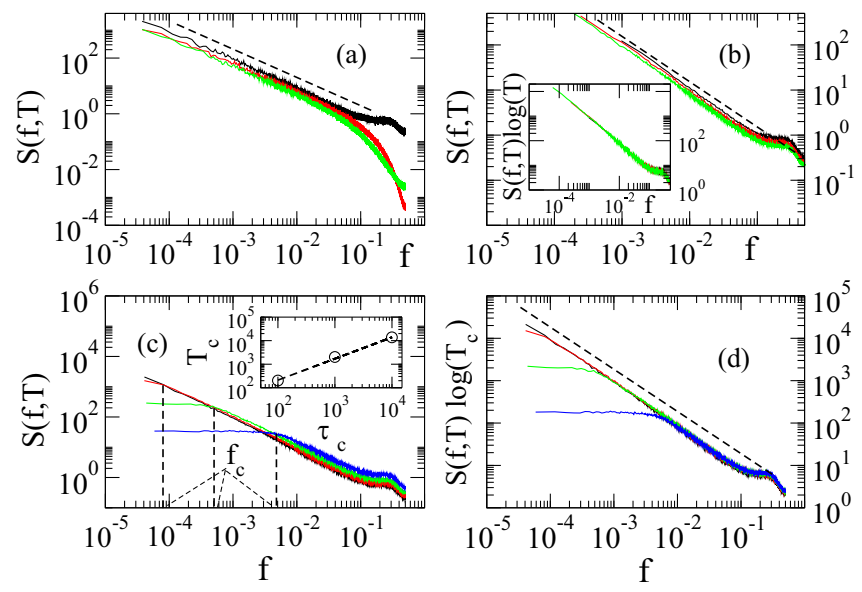

FIG. 1. PSDs of series of pulses with interpulse processes distributed following a power law $P(\tau) \sim \tau^{-2}$. (a) $T=2^{14}$ with exponential (green), Gaussian (red), and flat (black) pulses (hopping process). (b) Hopping process with distinct sizes, $T=2^{12}$ (black), $T=2^{14}$ (red), and $T=2^{16}$ (green). In the inset, collapse of spectra following the scaling law (1). (c) $T=2^{14}$ but now with truncated power laws with $\tau_{c}=10^{5}$ (black), $10^{4}$ (red), $10^{3}$ (green), and $10^{2}$ (blue). The inset shows the corresponding saturation sizes $T_{c}$. (d) Collapse of PSDs with truncated power laws following the scaling law (1) but now with $T_{c}$ instead of $T$. As an indication for the eye, dashed lines in (a), (b), and (d) follow an exact $1 / f$ law.

a series of pulses whose widths $\theta_{i}$ coincide with their interpulse times $\tau_{i}$. The spectral exponents can be calculated by doing $a=0$ in (4), as corresponds to pulses with constant amplitude, and $b=d$ in (4), because $\theta \equiv \tau$, giving

$$
\alpha_{s}=1-\frac{d}{2}, \alpha=0 .
$$

These are a special kind of process with very interesting properties that have been observed in several experiments with systems as quantum dots and liquid crystals [25]. In the classification by their spectral exponents [26], they belong to the stationary fractal (SF) class. They are stationary in a weak sense, since their variance is constant with the size $T$ ( $\alpha=0$ ) but their correlation depends not only on the time difference. They are fractals $\left(\alpha_{s}>0\right)$, but not self-affine $\left(\alpha_{s} \neq \alpha\right)$. Therefore, they do not have ergodic properties. Another interesting property is that their power spectra do not saturate at low frequencies, however they hold with the condition of finite total power, explaining the cutoff paradox of $1 / f$ noise [25,30]. In Fig. 1, a graphical representation of some of these properties is shown for the case $d=2$. Note that this is a limit case, $\alpha_{s}=0, \alpha=0$, which is in the frontier between being noise or fractal $\left(\alpha_{s}=0\right)$. It exhibits a perfect $1 / f$ shape, but, as indicated in the spectral scaling form (1), its spectrum has logarithmic corrections with $T$ as corroborated in Fig. 1(b).

Figure 1(a) shows that the shape of the pulse is irrelevant for low frequencies. The renewal process of Figs. 1(a) and 1(b) follows a complete power law while in those of Figs. 1(c) and $1(\mathrm{~d})$ the distribution is truncated at a given $\tau_{c}$. The effect of truncation, as shown in Figs. 1(c) and 1(d), is the appearance of a cutoff frequency $f_{c}$ in the spectra. The spectral scaling holds simply substituting the size $T$ with the corresponding cutoff 


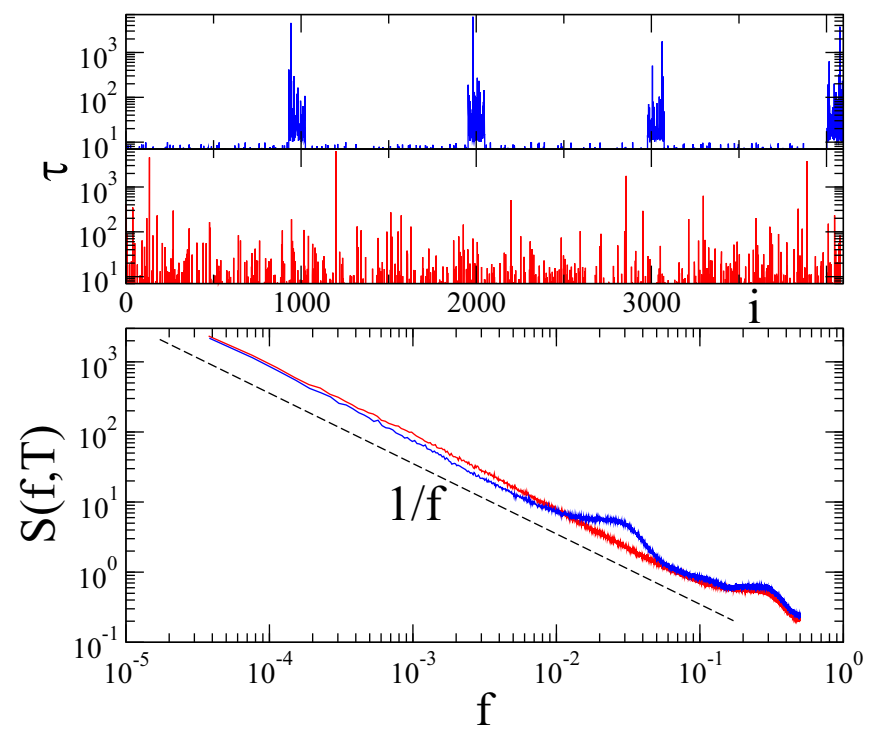

FIG. 2. Upper panel: Random (down, red) and correlated (up, blue) series of residence times distributed following a power law $P(\tau) \sim \tau^{-2}$. Lower panel: PSDs of the corresponding hopping processes averaged over 1000 samples. The $1 / f$ shape is robust against correlations.

time $T_{c}=1 / f_{c}$ [Fig. 1(d)]. This analysis is important because spectra obtained in real experiments usually show $1 / f$ shape in some range of frequencies. Below this range, saturation or crossover to other behaviors is possible. Note that a complete spectral analysis in this case involves sizes smaller than $T_{c}$, and in real situations this is usually a small range to find a scaling law. Then a joint analysis of a standard spectral method, giving the $\alpha_{s}$ exponent, with the distribution of residence times with a power law with exponent $-2\left(1-\alpha_{s}\right)$, would indicate that the process is in this class.

\section{Correlated pulses}

Typical series of correlated pulses that appear in the literature exhibit short-range inter-pulse distributions, usually Gaussian, with long-time correlations [26]. This kind of time series is classified, according to spectral properties, in the so-called class of pure $1 / f$ processes [26]. Their PSD presents a pure $1 / f$ shape $\left(\alpha_{s}=0\right)$ and the global exponent $\alpha$ is always negative and depending on the correlation. For a correlation of random walk type, $\alpha=-1 / 3$. These kinds of processes have been frequently used in the literature as models of systems whose fluctuations exhibit $1 / f$ noise [36]. However, they are not stationary and consequently they cannot be good models for many physical systems, including electronic and photonic devices. In fact, what we observe in our analysis of fluctuations in the intensity of VCSELs is that the origin of the observed $1 / f$ noise of our hopping process comes from other types of processes that are stationary (at least in a weak sense), $\alpha=0$, their residence time distribution follows a power law, and they are correlated forming clusters of uniform residence times.

For the sake of illustration, we plot in Fig. 2 two residence time series whose probability density follows a power-law distribution $P(\tau)=\tau^{-2}$, with $\tau>1$. One of the series has its times distributed randomly, while in the other, with the same values, they are grouped forming clusters. These clusters are formed separating in intervals residence times longer or shorter than one given threshold. Within each interval, the distribution of residence times is random. Besides the plot of series, we show the corresponding PSD of the normalized hopping process. We can see that the effect of the introduced quasiperiodic oscillations is the existence of a wide peak. But the important result is that the $1 / f$ original shape remains. So, one can expect that in a more general case, while correlations in the residence time series are of short range, the scaling of the uncorrelated case (6) continues being valid. A background line appears in the spectra given by this scaling with superimposed peaks accounting for the short-range correlation. This is what we are going to check in our signals.

\section{EXPERIMENTAL RESULTS}

A detailed description of the experimental setup used in this work can be found in [5]. In essence we have a tunable master laser that injects the parallel polarized light into the slave laser (VCSEL) through an optical circulator. The threshold current of the VCSEL at $298 \mathrm{~K}$ is $I_{\text {th }}=1.66 \mathrm{~mA}$, and this temperature is kept constant in the experiment, as is the bias current $I_{\text {bias }}=$ $5.0 \mathrm{~mA}$. In these conditions, the device emits in a linearly polarized single transverse mode at $\lambda=1541.82 \mathrm{~nm}$, with an orthogonal polarization mode, which is shifted by $33.36 \mathrm{GHz}$ toward the high frequencies with a side mode suppression ratio of $36 \mathrm{~dB}$. The optical injection is characterized by its strength $P_{i}$ and its frequency detuning $v_{i}$, defined as the difference between the frequency of the injected light and that of the free-running lasing mode. As shown in Fig. 2 of [7], several states involving the two linear polarization modes can be obtained for a fixed value of the detuning $\left(v_{i}=0.9 \mathrm{GHz}\right)$ and varying the injection level. Our experiment of hopping involves two states: one is a periodic dynamics (P1), similar to that illustrated in Figs. 2(a) and 2(b) of [7], while the other is a recently found state (IL+PS) [4], similar to the state shown in Figs. 2(e) and 2(f) of [7], that is a fixed point. Figure 3 shows the power of the orthogonal polarization signal for three different optical injection conditions. The P1 solution is characterized by oscillations of the power in the parallel polarization with a frequency close to the relaxation oscillation frequency while the power of the orthogonal polarization is constant and close to zero. In Fig. 3, only the orthogonal polarization power is shown, hence the P1 solution appears when the signal fluctuates around the zero value.

As is observed in Fig. 3, three kinds of hopping processes occur between these two states. In case (a), residence times of the P1 state $\left\{\tau_{1}\right\}$ exhibit strong variations. In the parameter space this corresponds to points close to the limit with the stability zone of $\mathrm{P} 1$. The same strong variation area is observed in case (c) but now for the IL+PS state. Parameters are now close to the limit with the stability zone of the IL+PS state. In (b), a standard behavior of a bistable system with noise is observed. The time residence distribution of both states is normal [7].

Concerning the coherence of signals, case (b) seems almost periodic, and then, assuming that fluctuations are driven by an external noise that cannot be controlled, we can say that this is a case of coherence enhancement. If one had control of the 

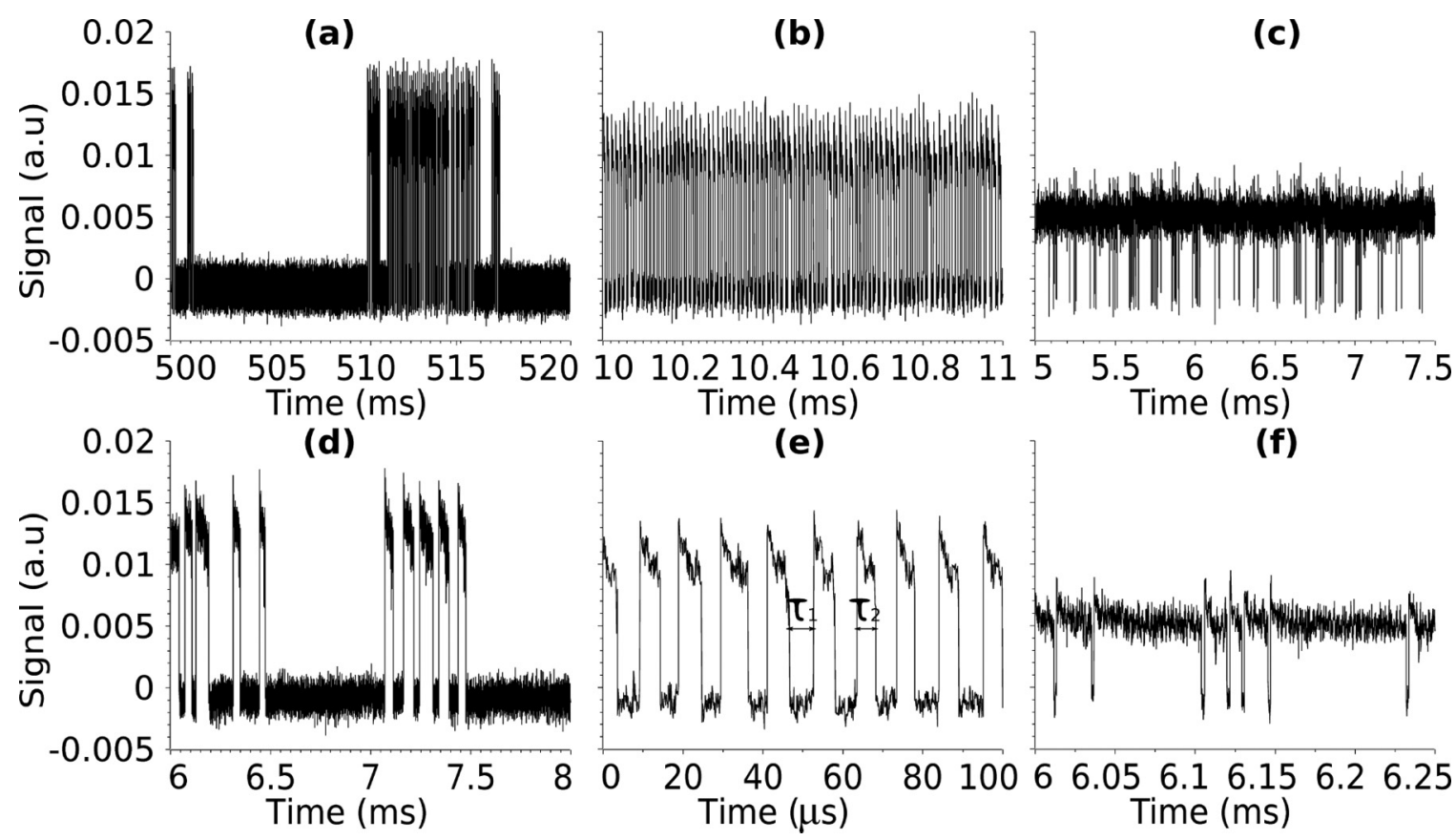

FIG. 3. Experimental time traces of the orthogonal polarization signal corresponding to the P1 \& IL+PS dynamics (a)-(c) and the zoomed parts of the time series (d)-(f). The system is in the P1 (IL+PS) state when the signal is small (large). The bias current is $I_{\text {bias }}=5 \mathrm{~mA}$. Left panel: $v_{i}=0.8 \mathrm{GHz}, P_{i}=48.01 \mu \mathrm{W}$. Center panel: $v_{i}=0.9 \mathrm{GHz}, P_{i}=53.83 \mu \mathrm{W}$. Right panel: $v_{i}=1.1 \mathrm{GHz}, \mathrm{P}_{\mathrm{i}}=64.71 \mu \mathrm{W}$.

noise intensity, it would be a case of coherence resonance [8]. The other two cases present a certain analogy with the on-off intermittent phenomenon [35] where the laminar state (off) is P1 or IL+PS, respectively, in (a) and (c).

To study the hopping process, it is convenient to assign mean values $I_{1}$ and $I_{2}$ to the intensity in each state, ignoring interstate fluctuations. Then we have a time series with values $I_{1}, I_{2}$ that can be represented by the series of residence times $\left\{\tau_{1 i}\right\}$ and $\left\{\tau_{2 i}\right\}$. Furthermore, since our analysis is based on spectral properties, we can just consider the normalized series $\left\{y_{i}=\frac{2}{I_{1}-I_{2}}\left(I_{i}-\frac{I_{1}+I_{2}}{2}\right)\right\}$, where the hopping states are, respectively, 1 and -1 and the spectra become multiplied by a constant value $S_{y}(f)=\frac{4}{\left(I_{1}-I_{2}\right)^{2}} S_{I}(f)$ with $f>0$. In this way, the spectral analysis is focused on residence times.

\section{HOPPING FLUCTUATIONS WITH INTERMITTENT BEHAVIOR}

The residence time distribution of $\tau_{2}$ for the injection conditions of Fig. 3(c), which is close to the region in which only IL+PS is stable, is shown in Fig. 4. We have obtained 24 consecutive 2.0 -s-long time traces with a $0.1 \mu$ s sampling time. The corresponding 24 residence time distributions are plotted with colored dashed lines while the overall distribution is plotted with diamonds. Close to the stability zones of the states P1 and IL+PS, the asymmetry of the residence times $\tau_{1}$ and $\tau_{2}$ is, respectively, very strong [7]. In fact, as shown in Fig. 4, the residence time of one of the states becomes distributed as a clear power law close to $\tau_{2}^{-2}$, whereas the other follows a non-heavy-tail distribution [Fig. 5(c)]. The result in the PSD of the hopping process is identical regardless of which state, the upper or lower, shows the heavy-tail distribution. For the sake of simplicity, we focus our analysis on case (c) of Fig. 3 always using a normalized description of the series. Moreover, we take as reference the exponent -2 in the power law of the distribution of residence times. Note that as shown in Fig. 4, this exponent is very close to -2 . The PSD in this case, as seen in Fig. 6, shows a background line with $1 / f$ shape in a determined range of frequencies and with a rich structure of peaks.

Our aim in this paper is to identify the class of our hopping process. Unfortunately, a complete spectral analysis is not

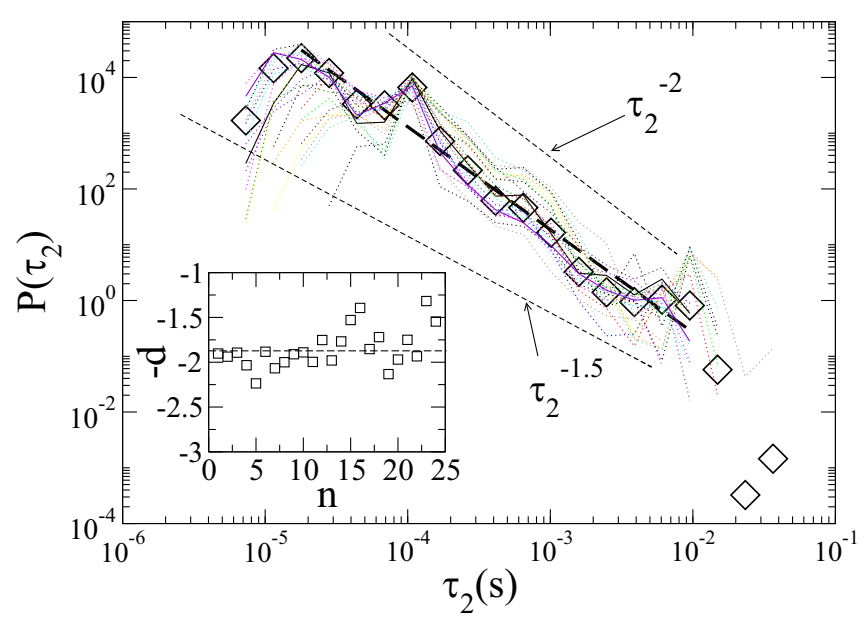

FIG. 4. Log-log plot of the probability density of experimental residence times $P\left(\tau_{2}\right)$ for each of the 24 samples (dot colored curves) and their average (diamond curve) with its fitted line in the interval $\left(10^{-5}, 10^{-2}\right) \mathrm{s}$ (thick dashed line) showing a decay, $P\left(\tau_{2}\right) \sim \tau_{2}^{-d}$, with $d=1.85$. Thin dashed lines are reference lines to guide the eye. Inset: fitted exponents of the probability density of each of the individual samples, giving $d=1.83 \pm 0.17$. 

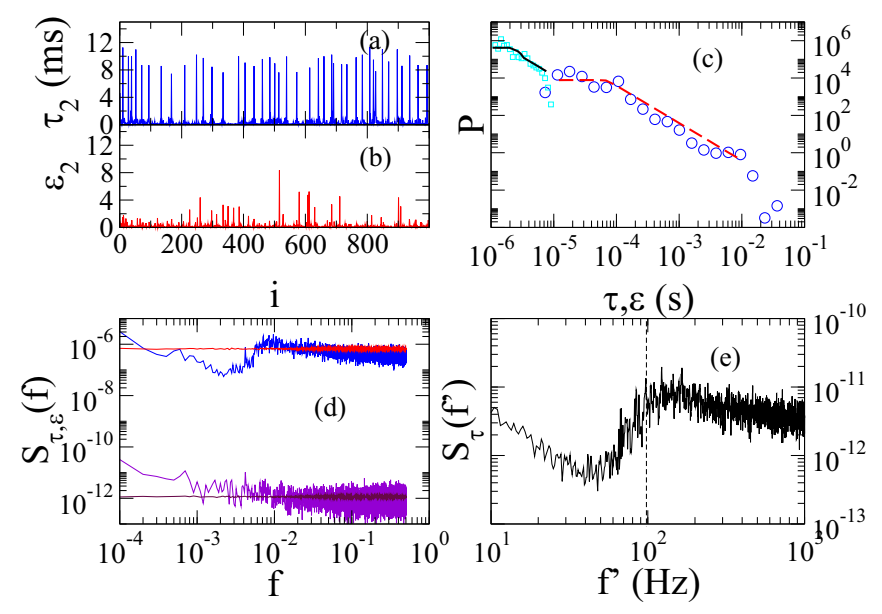

FIG. 5. (a) Residence time series $\left\{\tau_{2 i}\right\}$ of the hopping process observed in case (c) of Fig. 3, and (b) synthetically generated $\left\{\varepsilon_{2 i}\right\}$. (c) Probability density of the residence time series: $\tau_{2}$ (blue circles), $\tau_{1}$ (cyan squares), $\varepsilon_{2}$ (dashed line, red), and $\varepsilon_{1}$ (black continuous line). (d) Spectral densities of the four residence time processes, $S_{\tau_{2}}$ (upper, blue), $S_{\tau_{1}}$ (lower, violet), $S_{\varepsilon_{2}}$ (upper,red), and $S_{\varepsilon_{1}}$ (lower, brown). $S_{\tau}$ spectra are averaged over 24 samples while $S_{\varepsilon}$ spectra are averaged over 1000. (e) Detail of the peaked structure of the spectrum of $\tau_{2}$ in frequency units of $f^{\prime}=f /\langle\tau\rangle=f \times 3.7 \mathrm{kHz}$. The dashed line corresponds to the expected most prominent peak around $f=100 \mathrm{~Hz}$.

possible for two reasons. On the one hand, the frequency range in which the $1 / f$ shape is observed is too small and full of structural peaks that remain when the number of samples increases. On the other hand, the expected shift of spectra is logarithmic with the size $T$. Although in synthetic series this analysis is possible, as seen in Figs. 1(b) and 1(d), we have seen that, even with a great number of samples, the shift in the spectra cannot be confirmed. Therefore, the analysis

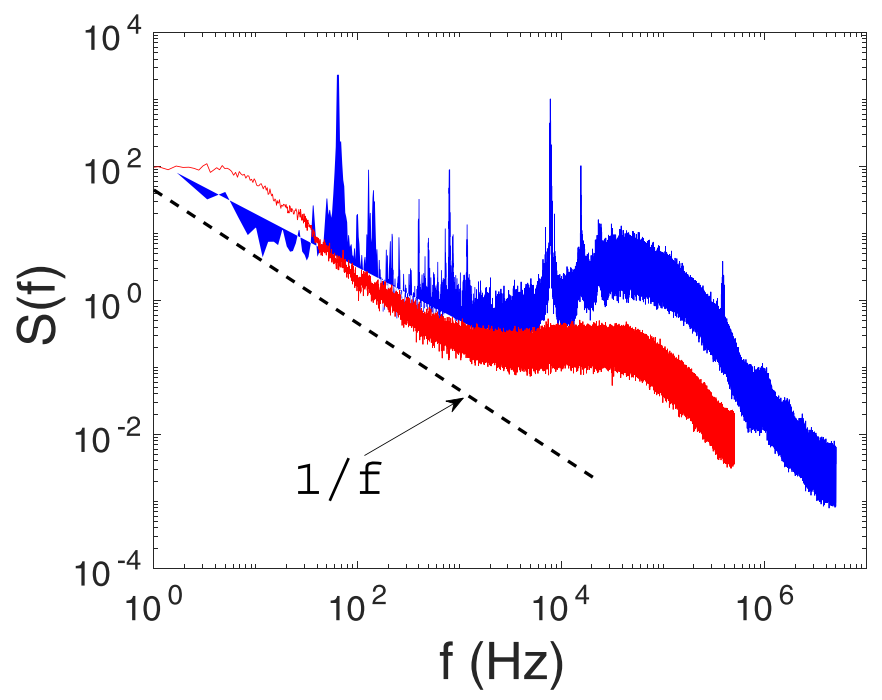

FIG. 6. PSD of the hopping process observed in the case (c) of Fig. 3 (blue spectrum) compared with that of a synthetically generated hopping process with the same distribution of residence times but in a random sequence (red spectrum). In both cases, the number of samples is 24 . should be performed with the local exponent of the spectra $\alpha_{s}$ and the exponent of the residence time distribution. In Fig. 5(a), we plot a typical series of residence times of the state with a power-law distribution $\left\{\tau_{2 i}\right\}$. In the same panel, for the sake of comparison, a time series with the same distribution but randomly sequenced is also plotted $\left\{\varepsilon_{2 i}\right\}$. The time series have the same probability density [see Fig. 5(c)] but different correlations. Note that the time residence series show clusters of high values similar to those depicted in Fig. 2 but with a richer structure. So, one can expect that, as occurs in this case, the spectra of the corresponding hopping processes keep their $1 / f$ shape, with a superimposed peaked structure as a result of correlations. To confirm this, we have compared the experimental series of the hopping process with others synthetically generated, keeping the same distribution in the residence times of both states, but with random and independent sequences. A graphical description of the results is shown in Fig. 5, where probability densities and spectra of residence times of experimental and synthetic series are plotted. For the generated series of residence times, we have used distributions that are uniform for a small first interval and power law with exponent -2 in the second one, fitting the experimental distribution, as shown in Fig. 5(c). Correlations are accounted for by means of PSDs [Fig. 5(d)]. Finally, the PSDs of the hopping process for the experimental and synthetic series are presented in Fig. 6.

Note that the differences between spectra are due to correlations between residence times of the same state and also between residence times of distinct states. The correlation index between time residence series of distinct states $\left\{\tau_{1 i}\right\}$ and $\left\{\tau_{2 i}\right\}(c=-0.89)$ is quite significant. It can affect the peaked structure, but it is irrelevant for the $1 / f$ shape. This is because one can consider pulses composed of two consecutive states forming steps, and take into account the fact that the shape of the pulses is irrelevant in the short frequency components of the spectral density.

Comparing the spectrum of residence times [Fig. 5(e)] with the spectrum of the hopping process (Fig. 6), one sees that the peaked structure becomes very amplified in this latter case. Note that the observed periodicity of around 40 units in the time residence series of Fig. 5(a) appears in the spectrum of Fig. 6 as a set of peaks around a frequency of $100 \mathrm{~Hz}$. Another prominent peak of $10^{4} \mathrm{~Hz}$ appearing in the spectrum of intensities (Fig. 6) is not visible in Figs. 5(d) and 5(e). This peaked structure with prominent peaks at $10^{2}$ and $10^{4} \mathrm{~Hz}$ is the signature of the external noise affecting our system.

\section{FLUCTUATIONS OF THE RESIDENCE TIME PROCESS IN THE COHERENCE ENHANCEMENT ZONE}

In the coherence enhancement zone, the residence time process of states $\left\{\tau_{1 i}\right\}$ and $\left\{\tau_{2 i}\right\}$ becomes similar. Now their probability densities do not follow a power law [Fig. 7(d)], but the spectrum of the residence times (Fig. 8) and the spectrum of the hopping process (Fig. 9) continue exhibiting $1 / f$ shape. So the origin of these fluctuations is now distinct from that observed in the preceding section. Note that, as shown in Fig. 8, all the unusual fluctuations that appear in the light intensity of the previous section now appear in the time residence process. The spectrum of residence times $S_{\tau}(f)$ that appears in Fig. 8 

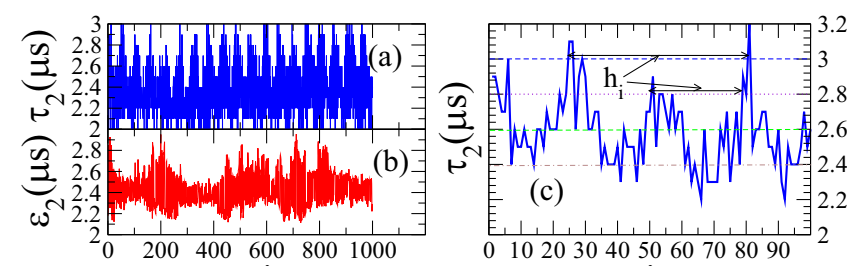

i
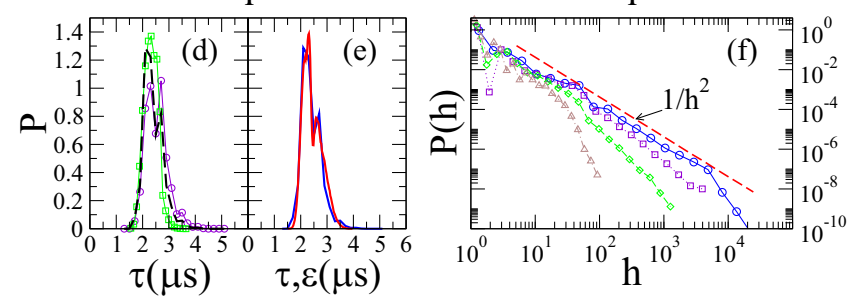

FIG. 7. (a) Time residence series $\left\{\tau_{2 i}\right\}$ of the hopping process observed in case (b) of our experiment. (b) Time residence series $\left\{\varepsilon_{2 i}\right\}$ obtained synthetically with the model shown in the text. (c) Method for obtaining the interpulse time series $\left\{h_{i}\right\}$ for a given threshold. (d) Probability density of residence times of each one of the states: $\tau_{2}$ (green squares), $\tau_{1}$ (violet circles), and their combined process (dashed black line). (e) Probability density of residence times of experimental (blue solid line) and synthetic (red solid line) signals. (f) Probability density of interpulse time series $\left\{h_{i}\right\}$ for several thresholds: 3.0 (blue circles), 2.8 (violet squares), 2.6 (green diamonds), and 2.4 (brown triangles).

is very distinct from the one shown in Figs. 5(d) and 5(e). In fact, it is very similar in shape to the spectrum of the hopping process in Fig. 6.

It would be interesting to know the origin of this behavior, but at this moment we do not have a proper model to explain it. What we can do is to classify the type of noise and understand why the $1 / f$ shape appears in the spectrum of the residence

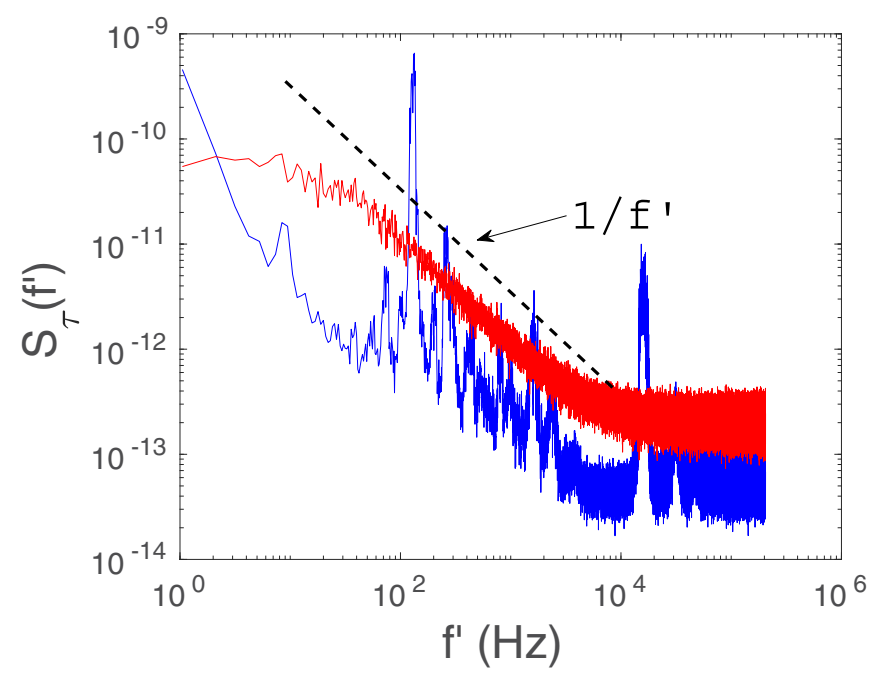

FIG. 8. PSDs of the time residence processes $\left\{\tau_{2}\right\}$ observed in the case (b) of Fig. 3 (blue spectrum) compared with that of a synthetically generated process with the same distribution of interpulse times but in a random sequence (red spectrum). To compare with PSDs of the hopping process, the frequency is taken as $f^{\prime}=f /\left\langle\tau_{2}\right\rangle=$ $0.4 \times f \mathrm{MHz}$. The number of samples is 24 in both cases.

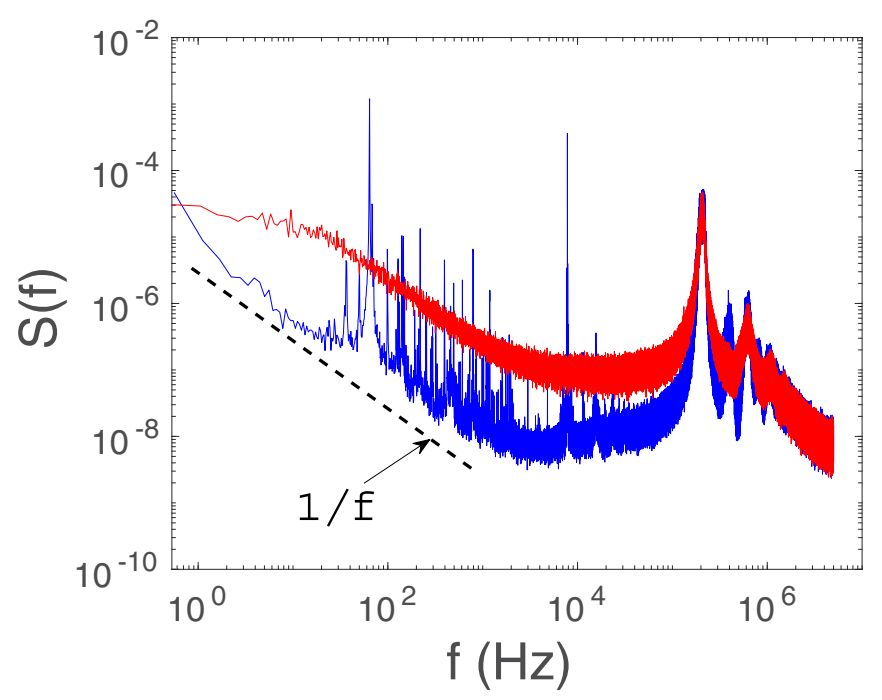

FIG. 9. PSDs of the hopping processes generated from the time residence series of the previous figure.

times $S_{\tau}(f)$. To this end, we first observe the shape of the time residence series of one of the states. Figure 7(a) shows that its behavior is completely distinct from that in the previous section. At short times one sees an alternating time series, and at longer times one sees a series of pulses. To get its interpulse time distribution, we take the intervals between the intersection of the series with a given threshold as illustrated in Fig. 7(c). The probability density of such intervals is plotted in Fig. 7(f). We can see a perfect power law, $P(h) \sim h^{-2}$, in up to four decades when taking the highest threshold. For lower thresholds the power law remains, but in fewer decades. Therefore, at this time scale we can say that this is a series of pulses whose interpulse distribution follows a power law with exponent -2 . And as we have shown, independently of the shape of pulses and correlations, the background line in the spectral density should exhibit a $1 / f$ shape.

To corroborate this result again, we have generated, as in the previous section, a synthetic time series with the same characteristics as the experimental series but in uncorrelated sequences. To emulate simultaneously the alternating behavior in short times with the pulse series observed at long times, we take two series of exponential pulses, $\left\{s_{i}^{+}\right\}$and $\left\{s_{i}^{-}\right\}$, with an intertime distribution given by the truncated power law of Fig. 7(f), one with larger and positive amplitude $A^{+}$and the other with smaller and negative amplitude $a^{-}$. The short behavior is accounted for by taking the values of one of the series with probability $p_{0}$ and adding a Gaussian random noise with intensity $D_{0}$. The obtained series $\left\{\varepsilon_{0 i}\right\}$ is finally adjusted to have the same spectral area doing the mean $\bar{\tau}$ and deviation $\sigma_{\tau}$ identical to those of the experimental series,

$$
\varepsilon_{i}=\frac{\sigma_{\tau}}{\sigma_{\varepsilon_{0}}}\left(\varepsilon_{0 i}-\overline{\varepsilon_{0}}\right)+\bar{\tau} .
$$

Simulations show that all synthetic series obtained in this way show a similar $1 / f$ shape in their spectra, since the relevant parameters are the exponent and cutoff of the interpulse time distribution. However, their probability densities $P(\varepsilon)$ are different. To get a probability density similar to the one 
obtained in experiments [see Fig. 7(e)], we have used the following values of the free parameters: $D_{0}=0.5, A^{+}=1$, $a^{-}=-0.25$. As shown in Fig. 8, the $1 / f$ shape in both spectra, experimental and synthetic, coincide in intensity and in frequency ranges. The peaked structure superimposed in the experimental spectrum is then due to correlations in the time residence process.

Finally, in Fig. 9 we plot the PSDs of their corresponding hopping processes. The origin of the $1 / f$ shape observed in the figure now comes from the correlation of the residence time series (Fig. 8), in clear contrast with the intermittent case, where it comes from the power-law character of their distribution. As in previous cases, synthetic series show the same $1 / f$ shape without a peaked structure. The upward shift of the spectra of synthetic series in the $1 / f$ zone of Fig. 8 is due to the assumption of equal variance between the experimental and synthetic series of residence times, which gives spectra with the same area. This shift is maintained in the spectra of hopping processes, as shown in Fig. 9.

\section{DISCUSSION AND CONCLUSIONS}

Light intensity fluctuations of VCSELs subject to parallel optical injection show an unusual behavior in some parameter space regions. We have analyzed the region in which two states, the so-called IL+PS, and one periodic process, P1, coexist. As a consequence of this coexistence, a light intensity hopping process between the two states is observed. Fluctuations of this process are analyzed either directly, by means of PSDs of the light intensity, or indirectly through the statistics of residence time series $\left\{\tau_{1 i}\right\}$ and $\left\{\tau_{2 i}\right\}$. Working in a broad range of frequencies between 1 and $10^{8} \mathrm{~Hz}$, we observe three clearly distinct processes. In one of the processes, the hopping is close to a process of coherence enhancement, in which the residence time series of the two states are statistically regular (not heavy tails). In the other two processes, away from this point, one of the residence time series, either $\left\{\tau_{1 i}\right\}$ or $\left\{\tau_{2 i}\right\}$, presents a power law in its distribution, and the PSD shows a $1 / f$ shape with a superimposed peaked structure. In the first case, the $1 / f$ shape is observed in both the PSD of the hopping process and also the PSD of the residence time process. To analyze these experimental results, some considerations of the known analytical theory have to be explored.

It is known that a hopping process whose residence time process is renewal and distributed according to a power law, $\tau^{-d}$, exhibits a PSD whose spectral exponent is determined by $d, \alpha_{s}=1-d / 2$. This result can be generalized to any series of pulses whose width is proportional to its interpulse time. In this paper, we address the question of how robust this scaling is against cutoffs in the distribution of residence (or interpulse) times, and short-range correlations of these times. We show how both effects affect the frequency range of the $1 / f$ shape and the existence of a peaked structure accounting for correlations, respectively. These results are very useful for analyzing real signals where both effects are usually present, as is our case.

With these results in mind, we conclude that the origin of the $1 / f$ shape observed in all cases is connected with the power law in the distribution of either residence times (in the case of intermittent behavior) or interpulses of the residence time series (in the coherence enhancement process). Our results show that we are in the presence of $1 / f$ noise of the SF class. To corroborate these results, we generate synthetic series with the same residence time (interpulse time) distributions but in random sequences, and we compare the obtained PSDs. A very similar $1 / f$ shape without a peaked structure is obtained, confirming our hypotheses.

It is worth remarking that there are several characteristics that differentiate our results from others with on-off intermittency in lasers. Our experiment shows a symmetry in the behavior between the two states IL+PS and P1 that does not correspond with the distinct character of both (fixed point and limit cycle). Furthermore, there are long intervals exhibiting power laws (up to three decades) in the residence time distributions and a value of exponents of these power laws close to -2 instead of the typical $-3 / 2$ observed in on-off intermittencies. So, although under these conditions we can observe a true on-off intermittency, that is, Hopf bifurcation driven by noise, the above-mentioned characteristics indicate that there is something more. The existence of a peaked structure in the PSD denotes the strong influence of external noise, but we do not have enough elements to explain this situation. Experiments with better control of the sources of noise could clarify this point, but this is beyond the scope of this work and it requires further investigation.

\section{ACKNOWLEDGMENT}

This work has been funded by the Ministerio de Economía y Competitividad (MINECO/FEDER, UE), Spain, under projects No. TEC2015-65212-C3-1-P (A. Valle) and No. Fis2016-74957-P (M. A. Rodríguez).
[1] K. Panajotov, I. Gatare, A. Valle, H. Thienpont, and M. Sciamanna, IEEE J. Quantum Electron. 45, 1473 (2009).

[2] X. Qi and J. M. Liu, IEEE J. Sel. Top. Quantum Electron. 17, 1198 (2011).

[3] Y. H. Hung, J. H. Yan, K. M. Feng, and S. K. Hwang, Opt. Lett. 42, 2402 (2017).

[4] A. Quirce, P. Pérez, A. Popp, A. Valle, L. Pesquera, Y. Hong, H. Thienpont, and K. Panajotov, Opt. Lett. 41, 2664 (2016).

[5] F. Denis-le Coarer, A. Quirce, A. Valle, L. Pesquera, M. Sciamanna, H. Thienpont, and K. Panajotov, Opt. Lett. 42, 2130 (2017).
[6] F. Denis-le Coarer, A. Quirce, P. Pérez, A. Valle, L. Pesquera, M. Sciamanna, H. Thienpont, and K. Panajotov, IEEE J. Sel. Top. Quantum Electron. 23, 1800910 (2017).

[7] F. Denis-le Coarer, A. Quirce, A. Valle, L. Pesquera, M. A. Rodriguez, K. Panajotov, and M. Sciamanna, Phys. Rev. E 97, 032201 (2018).

[8] A. Campos-Mejia, A. Pisarchik, R. Sevilla-Escoboza, G. HuertaCuellar, and V. Vera-Avila, Opt. Express 23, 10428 (2015).

[9] F. T. Arecchi and F. Lisi, Phys. Rev. Lett. 49, 94 (1982).

[10] M. R. Beasley, D. D'Humieres, and B. A. Huberman, Phys. Rev. Lett. 50, 1328 (1983). 
[11] M. Delgado-Restituto, A. Rodríguez-Vasquez, S. Espejo, and J. L. Huertas, IEEE Trans. Circ. Syst. 39, 325 (1992).

[12] F. T. Arecchi, R. Badii, and A. Politi, Phys. Rev. A 32, 402 (1985).

[13] B. Kaulakys, V. Gontis, and M. Alaburda, Phys. Rev. E 71, 051105 (2005).

[14] A. N. Pisarchik and V. J. Pinto-Robledo, Phys. Rev. E 66, 027203 (2002).

[15] G. Huerta-Cuellar, A. N. Pisarchik, and Y. O. Barmenkov, Phys. Rev. E 78, 035202(R) (2008).

[16] P. Manneville, J. Phys. (Paris) 41, 1235 (1980).

[17] T. Geisel, A. Zacherl, and G. Radons, Phys. Rev. Lett. 59, 2503 (1987).

[18] X. Brokmann, J. P. Hermier, G. Messin, P. Desbiolles, J. P. Bouchaud, and M. Dahan, Phys. Rev. Lett. 90, 120601 (2003).

[19] G. Margolin and E. Barkai, Phys. Rev. Lett. 94, 080601 (2005).

[20] L. Silvestri, L. Fronzoni, P. Grigolini, and P. Allegrini, Phys. Rev. Lett. 102, 014502 (2009).

[21] D. Krapf, Phys. Chem. Chem. Phys. 15, 459 (2013).

[22] T. Heil, I. Fischer, W. Elsasser, J. Mulet, and C. R. Mirasso, Opt. Lett. 24, 1275 (1999).
[23] S. Osborne, A. Amann, D. Bitauld, and S. O’Brien, Phys. Rev. E 85, 056204 (2012).

[24] A. Campos-Mejiaa, A. N. Pisarchik, and D. A. Arroyo-Almanza, Chaos Solitons Fractals 54, 96 (2013).

[25] M. Niemann, H. Kantz, and E. Barkai, Phys. Rev. Lett. 110, 140603 (2013).

[26] M. A. Rodríguez, Phys. Rev. E 90, 042122 (2014).

[27] N. Leibovich and E. Barkai, Phys. Rev. Lett. 115, 080602 (2015).

[28] A. Dechant and E. Lutz, Phys. Rev. Lett. 115, 080603 (2015).

[29] N. Leibovich, A. Dechant, E. Lutz, and E. Barkai, Phys. Rev. E 94, 052130 (2016).

[30] M. A. Rodríguez, Phys. Rev. E 92, 012112 (2015).

[31] J. W. Kantelhardt, E. Koscielny-Bunde, D. Rybski, P. Braun, A. Bunde, and S. Havlin, J. Geophys. Res. 111, D01106 (2006).

[32] W. Xiong, L. Faes, and P. C. Ivanov, Phys. Rev. E 95, 062114 (2017).

[33] G. W. Wornell, IEEE Trans. Inf. Theor. 36, 859 (1990).

[34] M. A. Rodríguez, Phys. Rev. E 95, 062309 (2017).

[35] P. W. Hammer, N. Platt, S. M. Hammel, J. F. Heagy, and B. D. Lee, Phys. Rev. Lett. 73, 1095 (1994).

[36] B. Kaulakys and G. Vektaris, Phys. Rev. E 52, 2091 (1995). 